\title{
Control of Photovoltaic Systems for Enhanced Short-Term Voltage Stability and Recovery
}

\author{
Gustav Lammert, Student Member, IEEE, Daniel Premm, \\ Luis David Pabón Ospina, Member, IEEE, Jens C. Boemer, Member, IEEE, \\ Martin Braun, Senior Member, IEEE, and Thierry Van Cutsem, Fellow Member, IEEE
}

\begin{abstract}
This paper investigates the impact of: i) the Low Voltage Ride-Through (LVRT) and Dynamic Voltage Support (DVS) capability; ii) the active current recovery rate; iii) the local voltage control; and iv) the plant-level voltage control of large-scale PhotoVoltaic (PV) systems on Short-Term (ST) voltage stability and Fault-Induced Delayed Voltage Recovery (FIDVR). Moreover, the influence on transient and frequency stability is studied briefly. To evaluate FIDVR, a novel metric, the socalled Voltage Recovery Index (VRI), is defined. The studies are performed with the WECC generic PV system model on an IEEE voltage stability test system, namely the Nordic test system. The results show that without LVRT capability the system is ST voltage and transient unstable. Only the LVRT and DVS capability help to avoid ST voltage and transient instability. Considering voltage and frequency dynamics, an active current recovery rate of $100 \% / \mathrm{s}$ shows the best performance. To further enhance voltage dynamics, plant-level voltage control together with local coordinated reactive power/voltage control should be applied. Moreover, the VRI provides useful information about the FIDVR and helps to compare different ST voltage controls.
\end{abstract}

Index Terms-Fault-induced delayed voltage recovery, dynamic reactive power support, dynamic grid support, fault ridethrough, induction motors, large-scale photovoltaic plants.

\section{INTRODUCTION}

\section{A. Motivation}

$\mathbf{T}$ HE electrical power system has undergone fundamental changes due to the increasing penetration of inverter based generation, i.e., wind and PhotoVoltaic (PV) generation. The dynamic characteristics of these technologies are different from conventional synchronous generators, which may impact the performance of the power system.

Gustav Lammert is with the Dept. of Energy Management and Power System Operation, University of Kassel, Kassel, Germany; e-mail: gustav.lammert@uni-kassel.de.

Daniel Premm is with the Dept. of Project Engineering, SMA Solar Technology AG, Niestetal, Germany; e-mail: daniel.premm@sma.de.

Luis David Pabón Ospina is with the Dept. of Power System Control and Dynamics, Fraunhofer Institute for Energy Economics and Energy System Technology (IEE), Kassel, Germany; e-mail: luis.david.pabon.ospina@iee.fraunhofer.de.

Jens C. Boemer is with the Dept. of Grid Operations and Planning, Modeling and Simulation, Electric Power Research Institute (EPRI), Palo Alto, USA; e-mail: jboemer@epri.com.

Martin Braun is with the Dept. of Energy Management and Power System Operation, University of Kassel, and the Dept. of Grid Planning and Operation, Fraunhofer Institute for Energy Economics and Energy System Technology (IEE), both in Kassel, Germany; e-mail: martin.braun@uni-kassel.de and martin.braun@iee.fraunhofer.de.

Thierry Van Cutsem is with the Fund for Scientific Research (FNRS) at the Dept. of Electrical Engineering and Computer Science, University of Liège, Liège, Belgium; e-mail: t.vancutsem@uliege.be.
Furthermore, recent incidents such as the Southern California event [1] or the South Australian blackout [2], both in 2016, highly motivate the studies reported in this paper. The former event was influenced by missing/incorrect Low Voltage Ride-Through (LVRT) requirements and too slow active current recovery rates [1]. The driving forces for the latter event were the inability to ride through multiple faults and the missing Dynamic Voltage Support (DVS) capability, i.e., no reactive current injection in response to the voltage dip [2]. Based on the recent work of the authors [3], the aforementioned aspects are addressed within this paper.

\section{B. Literature review}

A comprehensive review of power system stability challenges for large-scale PV integration is given in [4]. Recently, several studies have analyzed the impact of PV systems on transient stability [5]-[7], small-disturbance angle stability [6], [8] and frequency stability [7], [9]. Nevertheless, only a few studies have investigated the impact of PV systems on ShortTerm (ST) voltage stability and recovery, such as [10].

\section{Contributions}

In this paper, the impact of: i) the LVRT and DVS capability; ii) the active current recovery rate; iii) the local voltage; and iv) the plant-level voltage control of PV systems on ST voltage stability and recovery is reported. For this investigation, the WECC generic PV system model was considered and the studies were performed on the IEEE Nordic test system. The main contributions of this paper can be summarized as:

- a comprehensive analysis of different control methods of PV systems to ensure/improve ST voltage stability;

- a detailed investigation on how PV systems could enhance Fault-Induced Delayed Voltage Recovery (FIDVR) based on the WECC voltage criterion;

- a Voltage Recovery Index (VRI) to evaluate the phenomenon of FIDVR more systematically.

Other aspects are covered that lead to the following minor contributions:

- the influence of the LVRT and DVS capability of PV systems on transient stability;

- the impact of different active current recovery rates on frequency dynamics.

Finally, the paper gives recommendations for the adequate control of PV systems to improve the dynamic performance and avoid incidents such as those mentioned earlier. 


\section{Organization}

The remainder of the paper is organized as follows. Section II provides the definition of ST voltage stability considered in this paper. Section III outlines the load model and its composition. Section IV discusses the model structure and control of the PV systems. Section V presents the test system along with the modifications made to investigate ST voltage stability and recovery. Section VI details the metrics used to evaluate the impact of PV systems on power system dynamic performance. Section VII presents the numerical results. Finally, the conclusions and directions for future work are drawn in Section VIII.

\section{SHORT-TERM VOLTAGE STABILITY}

Voltage instability is a major threat in power system operation as it may trigger cascading failures and/or widespread blackouts. The time frame of interest for voltage stability problems may vary from a few seconds to tens of minutes. Therefore, voltage stability may be either a ST or a long-term phenomenon [11].

\section{A. Definition}

In general, ST voltage stability is defined according to the well-known report [11] as follows:

Short-term voltage stability involves dynamics of fast

acting load components such as induction motors,

electronically controlled loads, and HVDC converters.

In particular, ST voltage stability is understood in this paper as a phenomenon related to the stalling of Induction Motor (IM) loads, which try to restore their pre-disturbance power after a fault. For slowly cleared fault conditions IMs cannot reaccelerate, the mechanical and electromagnetic torque curves of the IM intersect, but at fault clearing the IM slip exceeds the unstable equilibrium value [12]. Therefore, the modelling of IMs is crucial.

\section{B. Analysis}

The study period of interest to analyze ST voltage stability is in the order of several seconds [11]. As ST voltage stability is a nonlinear stability problem [13], the analysis is based on solving differential algebraic equations of the system that capture these nonlinearities. In this study, the slips of IM loads are monitored via time-domain simulations to detect ST voltage instability. Transient P-V curves [10] or Lyapunov exponents [13] could be also applied. Further details to the evaluation methods are provided in Section VI.

\section{Related phenomena}

The phenomenon of FIDVR also plays a major role as it relates to the dynamic behavior of IM loads, which tend to decelerate following a large disturbance, resulting in low voltages in a significant portion of the power system. FIDVR can take place before ST voltage instability is reached. The time scale of ST voltage stability is also the time scale of transient (angle) stability. However, ST voltage instability should not be confused with the voltage drop that accompanies the loss of synchronism of a synchronous generator (transient instability). Both phenomena are also considered in this investigation.

\section{LOAD MODELLING}

As mentioned in Section II, accurate load modelling is important for the investigation of ST voltage stability and delayed voltage recovery. In the following sections, the composition of the aggregated load model is presented. The model includes a static and a dynamic part with a ratio of $70 \%$ and $30 \%$, respectively.

\section{A. Static part}

The static part is represented with an exponential load model [12] that is defined as:

$$
P=P_{0}\left(\frac{V}{V_{0}}\right)^{\alpha} \quad Q=Q_{0}\left(\frac{V}{V_{0}}\right)^{\beta}
$$

where $P$ and $Q$ are the active and reactive power, respectively, consumed by the load at the bus voltage $V . P_{0}$ and $Q_{0}$ are the active and reactive power, respectively, under the reference voltage $V_{0}$, obtained from the initial operating conditions. Since IMs are represented explicitly, the exponents of voltage dependency of active and reactive powers have been increased to $\alpha=\beta=2$ (i.e. a constant admittance is assumed).

\section{B. Dynamic part}

The dynamic part consists of an equivalent IM. This is a crucial component in ST voltage stability studies, because: i) it is a fast restoring load in the time frame of a second; ii) it is a low power factor load with a high demand of reactive power; and iii) it is prone to stalling, when voltage is significantly depressed [12]. In power system studies equivalent, aggregated IM models are usually considered. Therefore, the dynamic part of the load model consists of two equivalent, single-cage IMs, each with a third-order model. The IM model uses the rotor winding fluxes and the rotor speed as state variables. The IM rotates at a speed $\omega_{\mathrm{r}} \neq \omega_{\mathrm{s}}$ determined by the IM slip [12] given in:

$$
s=\frac{\omega_{\mathrm{s}}-\omega_{\mathrm{r}}}{\omega_{\mathrm{s}}}
$$

with the speed of the stator and rotor $\omega_{\mathrm{s}}$ and $\omega_{\mathrm{r}}$, respectively. The differential equation of the rotor motion dynamics [12] has the form of:

$$
2 H \frac{\mathrm{d} \omega_{\mathrm{r}}}{\mathrm{d} t}=T_{\mathrm{e}}\left(V, \omega_{\mathrm{r}}\right)-T_{\mathrm{m}}\left(\omega_{\mathrm{r}}\right)
$$

with the inertia constant $H$ and the electrical and mechanical torques $T_{\mathrm{e}}$ and $T_{\mathrm{m}}$, respectively. The mechanical torque is assumed constant as this is usually more critical for ST voltage stability:

$$
T_{\mathrm{m}}=T_{0}
$$

with $T_{0}$ as the torque value determined from the initial power flow computation, assuming the motor is operating in steady state. The $30 \%$ IM load is sub-divided into $15 \%$ of small and $15 \%$ of large industrial motors with power factors of 0.6 and 0.8 , respectively [14]. Finally, a compensation shunt capacitor is connected in parallel to the IM in order to match the reactive power consumption of the entire (static and dynamic) load in steady state. 


\section{Photovoltaic SySTEM MODELLING}

\section{A. Generic model}

The large-scale generic PV system model used to study the impact on ST voltage stability and recovery follows the WECC specifications [15], [16] and was implemented and validated in [17]. An overview of the WECC generic PV system model is given in Fig. 1 and it consists of the Renewable Energy Generator/Converter (REGC_A) model, the Renewable Energy Electrical Control (REEC_B) model and the Renewable Energy Plant Control (REPC_A) model, which are interfaced with each other. Different control modes of the PV system, i.e., parameter settings of the REGC_A, REEC_B and REPC_A model shown in Figs. 2, 3 and 4, respectively, are investigated and the relevant parameters are highlighted in green. These variations represent presently required operating modes and settings according to international grid codes and the parameter set is nearly similar to [3]. Reactive current priority is considered in all modes. The different controllers have an impact on different dynamics, i.e., the REGC_A and REEC_B model affect dynamics in the order of a few seconds, while the REPC_A model affects dynamics in the order of several seconds up to minutes. An overview of the equivalent system is given in Fig. 5. The investigation focuses on largescale PV plants connected to the high- or extra-high-voltage level. The voltage is measured and controlled at the Point of Common Coupling (PCC), as shown in Fig. 5. Note that only the positive sequence control of PV systems is of interest in this investigation.

\section{B. LVRT and DVS capability}

The following control modes are considered for the response of the PV system to faults and the corresponding parameter variations are defined in REGC_A_1 of Fig. 2 and REEC_B_1 of Fig. 3.

1) No LVRT capability (No LVRT): In this mode the undervoltage protection immediately trips the PV system in response to the voltage dip and the plant is merely disconnected. This setting represents old grid code requirements in Germany.

2) LVRT with blocking mode (LVRT \& block): In this mode the PV system remains connected to the grid but it does not inject any active or reactive current during the fault-on period. This mode is also called zero power or momentary cessation mode and is apparently applied in many inverters in USA [1]. By activating the low voltage power logic Lvplsw $=1$ in REGC_A_1 of Fig. 2, and setting the static gain $K q v=0$ in REEC_B_1 of Fig. 3, this mode is enabled.

3) LVRT with DVS (LVRT \& DVS): This control mode corresponds to the requirements of the recent German grid code for high-voltage networks [18]. Similarly to the former control mode, the PV system remains connected to the grid during the fault (LVRT requirement). However, in order to support the voltage during the disturbance, an additional reactive current is injected, also called DVS. The additional reactive current is calculated as $i q i n j=K q v \cdot \Delta V$ with the static gain $K q v$ and the voltage deviation $\Delta V=V r e f 0-V t \_f i l$ determined from the pre-fault condition as seen in REEC_B_1 of Fig. 3. The static gain is set to $K q v=4$.

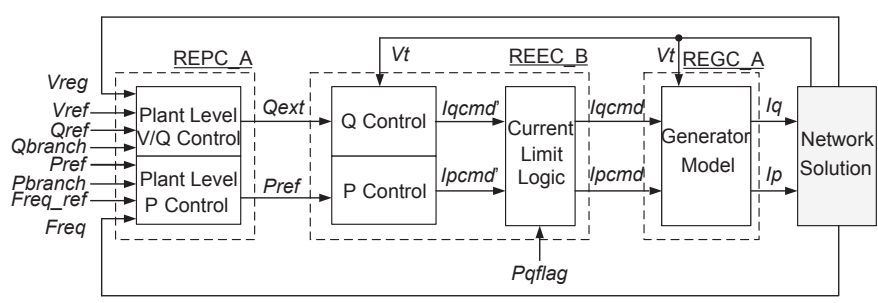

Fig. 1. Control block diagram of the WECC generic PV system model [15].

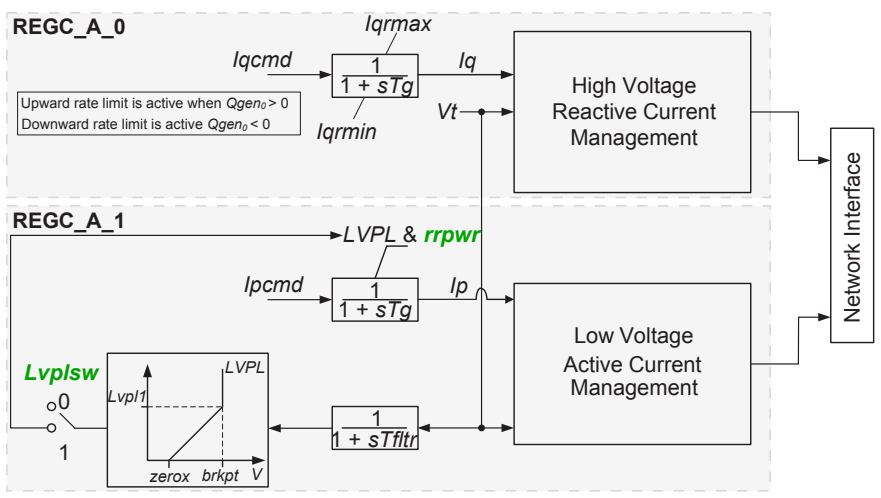

Fig. 2. Control block diagram of the REGC_A model [15].

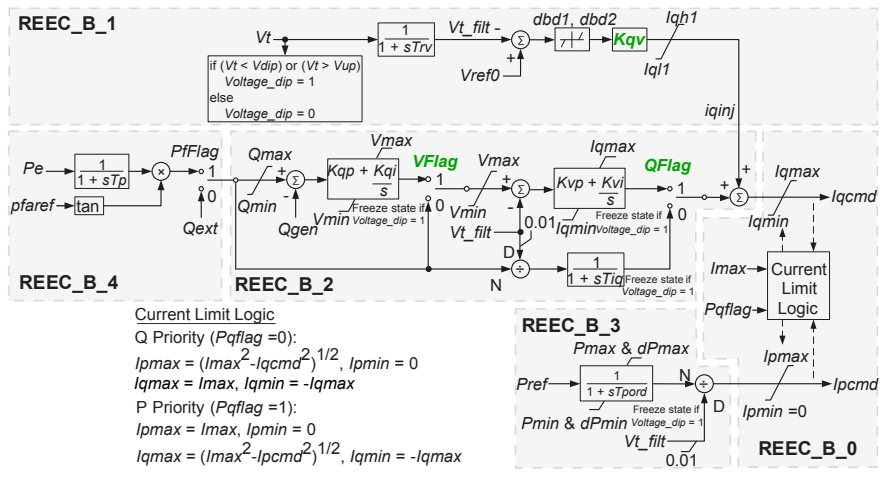

Fig. 3. Control block diagram of the REEC_B model [15].

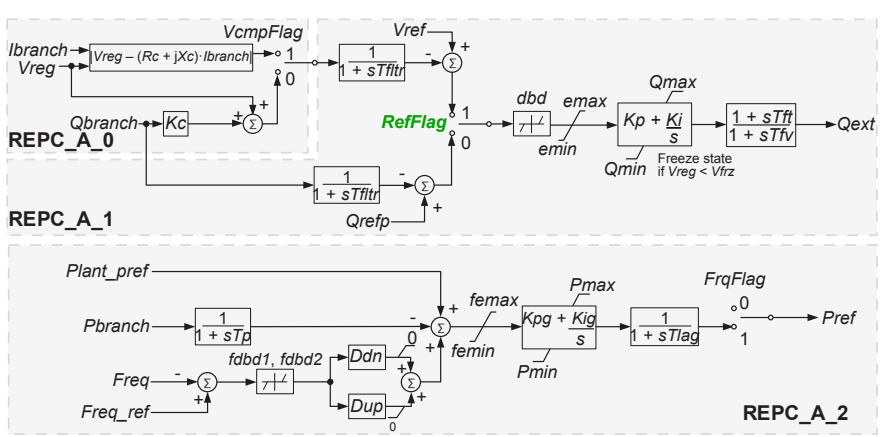

Fig. 4. Control block diagram of the REPC_A model [15].

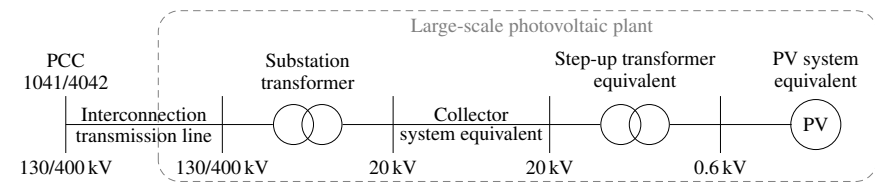

Fig. 5. Single-generator equivalent system of the large-scale PV plant. 


\section{Active current recovery rate}

The following control modes relate to the variation of the active current recovery rate rrpwr, during the post-fault period, as shown in REGC_A_1 of Fig. 2.

1) Very slow recovery $(10 \% / s)$ : This setting represents a recovery rate with $\mathrm{rrpwr}=10 \% / \mathrm{s}$, which is widely used in USA [1].

2) Slow recovery $(20 \% / s)$ : This setting describes a recovery rate with $r r p w r=20 \% / \mathrm{s}$, which is the lower limit defined by the German grid code [18].

3) Medium recovery $(100 \% / s)$ : This setting defines a recovery rate with $r r p w r=100 \% / s$, which is the lower limit defined by the grid code in Great Britain for faults with a duration of more than $140 \mathrm{~ms}$ [19].

4) Fast recovery $(1000 \% / s)$ : This setting specifies a recovery rate with $r r p w r=1000 \% / s$, which is the lower limit defined by the grid code in Great Britain for faults with a duration of less than $140 \mathrm{~ms}$ [19].

\section{Local voltage control}

The subsequent modes refer to different local voltage control strategies during normal operation $\left(V t \_f i l t>0.9 \mathrm{pu}\right.$ ) that can be set in REEC_B_2 of Fig. 3 in order to investigate the influence on ST voltage stability and recovery. The corresponding flag combinations are shown in Table I.

1) Local constant reactive power control (Local $Q$ ctr): This control mode uses the feed-forward loop in REEC_B_2 with $Q F l a g=0$. The reactive power is kept at the value given by the initial power flow computation.

2) Local constant voltage control (Local V ctr): This mode enables constant voltage control by using the second PI controller in REEC_B_2 with QFlag $=1$ and VFlag $=0$ that keeps the voltage at its initial power flow value.

3) Local coordinated reactive power/voltage control (Local $Q / V$ ctr): This control mode allows a coordinated reactive power/voltage control by using the two PI controllers in series in REEC_B_2 of Fig. 3 with QFlag = VFlag $=1$. The controllers allow a fast voltage control to restore the voltage quickly after the fault, followed by a slow reactive power control that brings the PV system back to its reactive power set point in steady state.

\section{E. Plant-level voltage control}

The following modes involve the plant-level control, i.e., the REPC_A model shown in Fig. 4, to study its impact on the dynamics. The corresponding flag combinations are shown in Table II. For all subsequent plant-level control modes, reactive power limits of $Q \max =0.4 \mathrm{pu}$ and $Q \min =-0.4 \mathrm{pu}$ on the PV system MVA base are imposed in REPC_A_1, as shown in Fig. 4. It should be noted that compared to the local control in Section IV-D, the plant control has a slower response time and thus influences system dynamics in the order of several seconds up to minutes. A typical example of plant control is the provision of frequency response [9].

1) Plant-level reactive power control (Plant $Q$ ctr): This mode allows constant reactive power control using the lower path in REPC_A_1 of Fig. 4 with RefFlag $=0$ and the feedforward loop in REEC_B_2 of Fig. 3 setting QFlag $=0$.
TABLE I

LOCAL VOLTAGE CONTROL MODES

\begin{tabular}{|l|c|c|}
\hline Mode of operation & VFlag & QFlag \\
\hline \hline Local Q ctr & 0 or 1 & 0 \\
\hline Local V ctr & 0 & 1 \\
\hline Local Q/V ctr & 1 & 1 \\
\hline
\end{tabular}

TABLE II

Plant-leVel Voltage CONTROL MOdES

\begin{tabular}{|l|c|c|c|}
\hline Mode of operation & VFlag & QFlag & RefFlag \\
\hline \hline Plant Q ctr & 0 or 1 & 0 & 0 \\
\hline Plant V ctr & 0 or 1 & 0 & 1 \\
\hline Plant Q ctr \& Q/V ctr & 1 & 1 & 0 \\
\hline Plant V ctr \& Q/V ctr & 1 & 1 & 1 \\
\hline
\end{tabular}

2) Plant-level voltage control (Plant V ctr): This mode enables constant voltage control using the upper path in REPC_A_1 of Fig. 4 with RefFlag $=1$ and the feed-forward loop in REEC_B_2 of Fig. 3 with QFlag $=0$.

3) Plant-level reactive power control and local coordinated reactive power/voltage control (Plant $Q$ ctr \& $Q / V$ ctr): This control mode is a combination of plant-level constant reactive power control in REPC_A_1 of Fig. 4 with RefFlag $=0$, together with local coordinated reactive power/voltage control in REEC_B_2 of Fig. 3 with VFlag $=$ QFlag $=1$.

4) Plant-level voltage control and local coordinated reactive power/voltage control (Plant $V$ ctr \& $Q / V$ ctr): The last mode is a combination of plant-level constant voltage control in REPC_A_1 of Fig. 4 with RefFlag = 1, together with local coordinated reactive power/voltage control in REEC_B_2 of Fig. 3 with VFlag $=$ QFlag $=1$.

\section{TEST SYSTEM}

\section{A. Overview}

The power system dynamic performance including largescale PV plants has been investigated using the IEEE Nordic test system detailed in [20], which was implemented and validated in DIgSILENT PowerFactory, as reported in [21]. The single-line diagram is shown in Fig. 6. The operating point $\mathrm{B}$ is considered, as documented in [20].

\section{B. Integration of $P V$ systems}

The initial system configuration is without PV systems and serves as the base case. In the next step, synchronous generators are replaced with large-scale PV systems as follows: g6 and g7 by 5 PV plants (130 MVA each) connected to buses 1041 to 1045 ; g14 by 3 PV systems (240 MVA each) connected to buses 4042, 4043 and 4046; and g17 by 2 PV plants (300 MVA each) connected to buses 4061 and 4062, as seen in Fig. 6. Table III gives the active power load, generation and the penetration level in each area and for the entire system. The PV penetration level is calculated as the PV generation divided by the total generation. In order to match operating point B as in [20], a new shunt is connected to bus 1042 and the other existing shunts are adjusted. All PV plants consist of the single-generator equivalent system shown in Fig. 5. They are connected to the high- or extra-high-voltage level in the test system (see Fig. 6), and operated at unity power factor. 


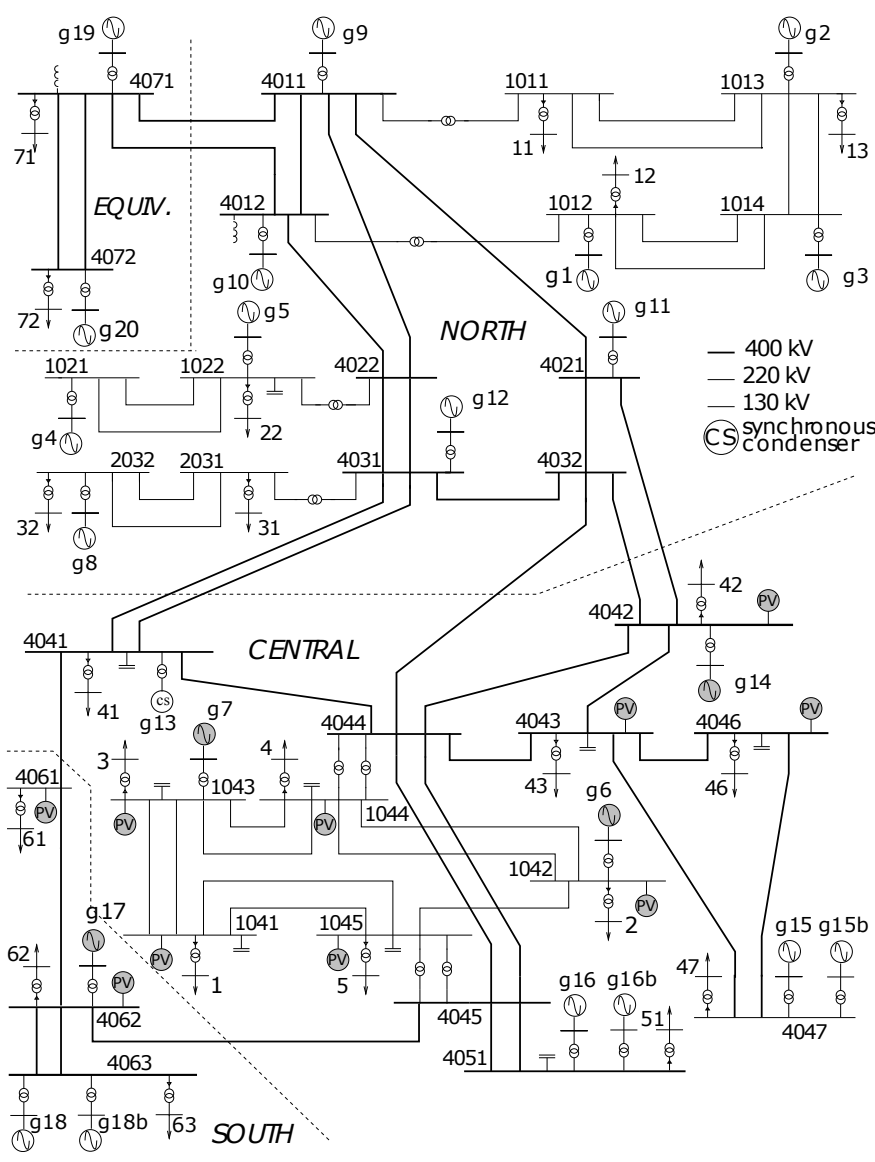

Fig. 6. Nordic test system with large-scale PV plants based on [20].

TABLE III

ACTIVE POWER LOAD AND GENERATION OF THE NORDIC TEST SYSTEM

\begin{tabular}{|c|c|c|c|c|c|}
\hline \multirow{2}{*}{ Area } & \multicolumn{2}{|c|}{ Generation [MW] } & \multicolumn{2}{c|}{ Load [MW] } & \multirow{2}{*}{ Penetration [\%] } \\
\cline { 2 - 5 } & Sync. gen. & PV & Stat. & Dyn. & \\
\hline \hline North & 4628.5 & 0 & 826 & 354 & 0 \\
\hline Central & 1680 & 1170 & 4333 & 1857 & 41 \\
\hline South & 1050 & 540 & 973 & 417 & 34 \\
\hline Equiv & 2437.4 & 0 & 2300 & 0 & 0 \\
\hline \hline Total & 9795.9 & 1710 & 8432 & 2628 & 15 \\
\hline
\end{tabular}

\section{EVALUATION OF POWER SYSTEM DYNAMIC PERFORMANCE}

\section{A. Time-domain simulation}

The results were obtained from time-domain (phasor mode) simulations performed with DIgSILENT PowerFactory [22]. The integration time step is $0.01 \mathrm{~s}$.

\section{B. Critical Clearing Time (CCT)}

The Critical Clearing Time (CCT) is the maximum permissible duration of the fault for which the subsequent system response remains stable [11]. In this study, the CCT is determined as the longest duration of the fault that does not cause:

- stalling of any IM load (ST voltage instability);

- loss of synchronism of any synchronous generator (transient instability).
If only one of these conditions holds true, the fault duration exceeds the CCT. In general, the longer the CCT, the better the dynamic performance of the power system. It should be noted that the CCT is usually applied to assess transient stability [6], [7]. However, it has been also used to evaluate ST voltage stability [10].

\section{Voltage Recovery Index (VRI)}

Available methods in literature to quantify FIDVR can be classified into two categories [23]:

- Slope-based methods: metrics based on the slope or the derivative of the voltage evolution are not the most appropriate in case of oscillations or sudden (discontinuous) changes in the voltage.

- Integral error-based methods: those techniques cannot distinguish between the following two voltage waveforms: i) one with a lower initial voltage drop that takes more time to recover, and ii) the other with a higher initial voltage drop but showing fast recovery over a small period of time.

To deal with the aforementioned limitations, the KullbackLeibler (KL) divergence measure was introduced in [23]. However, the authors of this paper met several issues using the KL measure for the evaluation of FIDVR. The calculation of the KL measure as well as its limitations are detailed in the Appendix. These limitations motivated the authors to define a new metric, namely the VRI, to evaluate the phenomenon of FIDVR more systematically. The steps involved in calculating the VRI for a specific voltage waveform are as follows.

1) Voltage violation criterion: A voltage violation criterion is defined that determines the lowest acceptable voltage. In this work, the WECC voltage violation criterion according to [24] is considered, which has been used in several studies [23], [25] to evaluate FIDVR. An example of the WECC voltage violation criterion along with two sample voltage recoveries is illustrated in Fig. 7 (a). The parameters $V_{1}, V_{2}, V_{3}$ and $t_{1}, t_{2}$ denote the voltage and time limits, respectively, and are shown in Table IV.

2) Probability density function of voltage sample: If the voltage recovers quickly to its steady-state value, the corresponding Probability Density Function (PDF) has a high peak near that steady-state value. The opposite holds true when the voltage recovery is slow. To define the PDF for the VRI, the voltage axis from $V_{\min }$ to $V_{\max }$ is partitioned into $L$ subintervals, where the index $i$ refers to the $i$-th partition and the values are given in Table IV. The voltage samples are observed from the fault clearing instant $t_{\mathrm{cl}}$ to the final observation time

TABLE IV

VOLTAGE RECOVERY INDEX PARAMETERS

\begin{tabular}{|c|c|c|c|c|c|}
\hline$V_{\min }[\mathrm{pu}]$ & $V_{1}[\mathrm{pu}]$ & $V_{2}[\mathrm{pu}]$ & $V_{3}[\mathrm{pu}]$ & $V_{\max }[\mathrm{pu}]$ & $t_{\mathrm{cl}}[\mathrm{s}]$ \\
\hline 0.0 & 0.75 & 0.8 & 0.95 & 1.2 & 0.1 \\
\hline \hline$t_{1}[\mathrm{~s}]$ & $t_{2}[\mathrm{~s}]$ & $t_{\mathrm{f}}[\mathrm{s}]$ & $\lambda_{1}^{+}[-]$ & $\lambda_{2}^{+}[-]$ & $\lambda_{3}^{+}[-]$ \\
\hline 0.8 & 2.5 & 3.0 & 70 & 150 & 3000 \\
\hline \hline$\lambda_{1}^{-}[-]$ & $\lambda_{2}^{-}[-]$ & $\lambda_{3}^{-}[-]$ & $L[-]$ & $\sigma[-]$ & - \\
\hline 11 & 8 & 5 & 100 & 0.01 & - \\
\hline
\end{tabular}




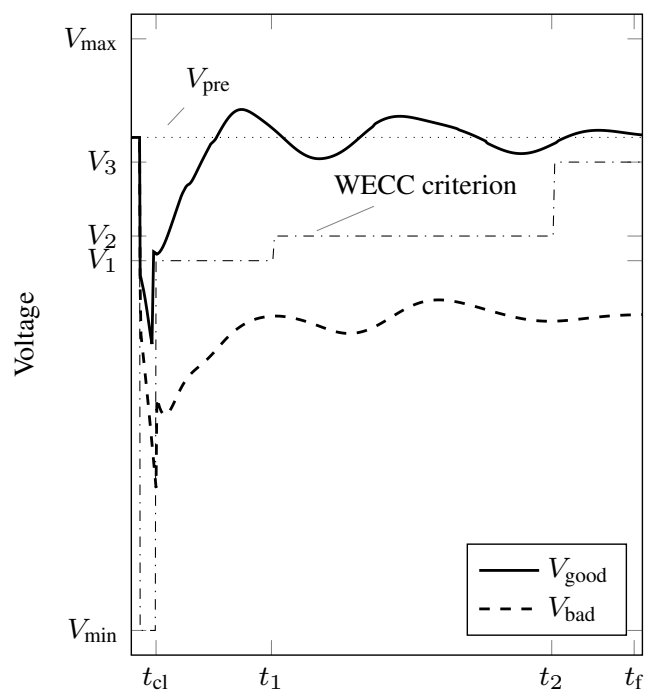

(a)

Time

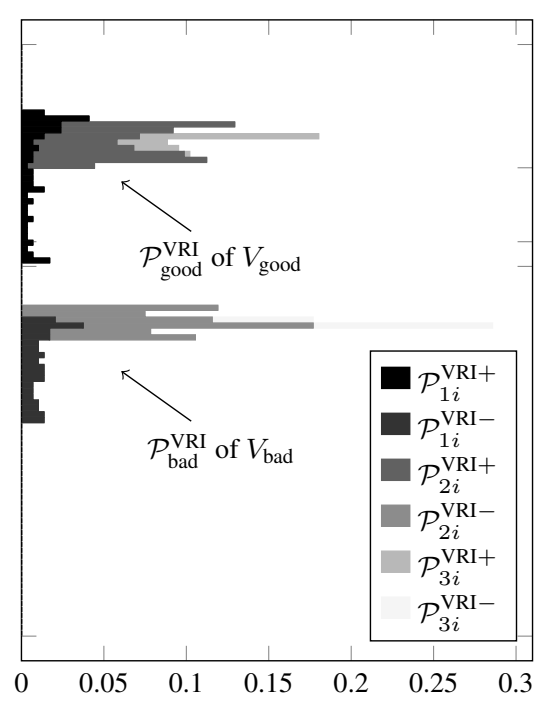

(b) Probability density functions

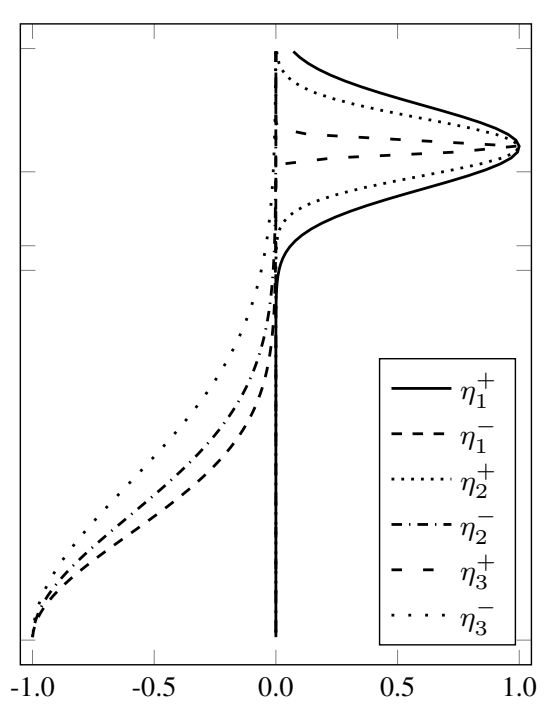

(c) Weighting functions

Fig. 7. Basic procedure to determine the Voltage Recovery Index (VRI). (a) Voltage violation criterion (WECC criterion) and sample voltage recoveries. (b) Probability Density Functions (PDFs) of sample voltage recoveries. (c) Weighting functions that reward or penalize the voltage recovery.

$t_{\mathrm{f}}$, as also given in Table IV. Considering the WECC voltage violation criterion and a given voltage time series $V(t)$, the rewarding $\mathrm{PDF} \mathcal{P}_{i}^{\mathrm{VRI}+}$ and the penalizing $\mathrm{PDF} \mathcal{P}_{i}^{\mathrm{VRI}-}$ are constructed, as shown in the algorithm of Fig. 8. The number of samples in a particular sub-interval $\mathcal{P}_{i}^{\mathrm{VRI}}$ is divided by the total number of samples $A$ to obtain a normalized distribution. Hence, the overall PDF $\mathcal{P}^{\mathrm{VRI}}$ is:

$$
\mathcal{P}^{\mathrm{VRI}}=\frac{1}{A} \sum_{j=1}^{M} \sum_{i=1}^{L}\left(\mathcal{P}_{j i}^{\mathrm{VRI}+}+\mathcal{P}_{j i}^{\mathrm{VRI}-}\right)
$$

where $M$ denotes the number of voltage constraints and the index $j$ refers to the $j$-th voltage constraint. It should be emphasized that compared to the PDF for the KL divergence measure $\mathcal{P}^{\mathrm{KL}}$ given in (10) (see Appendix), the PDF for the VRI $\mathcal{P}^{\text {VRI }}$ defined in (5) does not only give information about the distribution of the voltage, but it also shows its relation to the constraints (if voltage limits are violated) and the times (when voltage limits are violated). The specific PDF with regard to the WECC criterion, for instance, consists of six PDFs in total, two according to the voltage violations (i.e. $V_{1}, V_{2}, V_{3}$ ) times three according to the time intervals (i.e. $\left[t_{\mathrm{cl}}, t_{1}\left[;\left[t_{1}, t_{2}\left[;\left[t_{2}, t_{\mathrm{f}}[)\right.\right.\right.\right.\right.$, as depicted in Fig. 7 (b).

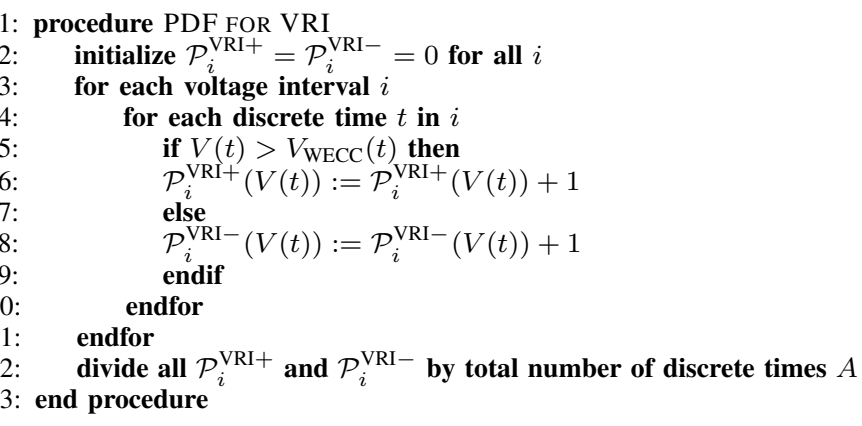

Fig. 8. Algorithm for the construction of the probability density function.
3) Weighting function: To reward (+) or penalize (-) the voltage recovery, weighting functions are applied:

$$
\begin{gathered}
\eta_{j}^{+}=\mathrm{e}^{-\lambda_{j}^{+}\left(V_{i}-V_{\text {pre }}\right)^{2}} \text { for } j=1, \ldots, M \text { and } i=1, \ldots, L \\
\eta_{j}^{-}=-\mathrm{e}^{-\lambda_{j}^{-}\left(V_{i}-0\right)^{2}} \text { for } j=1, \ldots, M \text { and } i=1, \ldots, L
\end{gathered}
$$

where the parameter $\lambda$ controls the width. The values of $\lambda$ significantly influence the resulting VRI and should be tuned according to the violation criterion, i.e., in the case of $V_{\mathrm{WECC}}$ :

$$
|\eta|<\sigma \quad \text { for } \quad V_{i}=V_{\mathrm{WECC}}
$$

Considering the WECC criterion with $M=3$, the so defined weighting functions are depicted in Fig. 7 (c). The corresponding values for $\lambda_{j}^{+}, \lambda_{j}^{-}$, and $\sigma$ are listed in Table IV.

4) Calculation of index: Finally, the VRI, denoted as $\mathcal{V}$, is calculated using the PDFs and the weighting functions:

$$
\mathcal{V}=\frac{1}{A} \sum_{j=1}^{M} \sum_{i=1}^{L}\left(\eta_{j i}^{+} \mathcal{P}_{j i}^{\mathrm{VRI}+}+\eta_{j i}^{-} \mathcal{P}_{j i}^{\mathrm{VRI}-}\right)
$$

The lower bound of the VRI corresponds to no voltage recovery $(V=0 \mathrm{pu})$, leading to $\mathcal{V}=-1$. The upper bound of the VRI is reached for an ideal immediate recovery ( $\left.V=V_{\text {ideal }}\right)$, leading to $\mathcal{V}=+1$. These clear bounds of the VRI give another advantage of this metric compared to the KL measure. Moreover, the VRI is "neutral" if the voltage recovers exactly to the specified voltage violation criterion. It can be concluded that the higher the value of $\mathcal{V}$, the better the voltage recovery. The obtained VRIs for the sample voltage recoveries in Fig. 7 (a) are shown in Table V.

TABLE V

VOLTAGE RECOVERY INDICES

\begin{tabular}{|c|c|c|}
\hline $\mathcal{V}_{\text {good }}$ of $V_{\text {good }}[-]$ & $\mathcal{V}_{\text {WECC }}$ of $V_{\text {WECC }}[-]$ & $\mathcal{V}_{\text {bad }}$ of $V_{\text {bad }}[-]$ \\
\hline 0.79 & 0.00 & -0.06 \\
\hline
\end{tabular}




\section{CASE STUDIES}

\section{A. Overview}

An overview of all the reported case studies is given in Table VI. The initial system configuration is without PV systems and serves as a reference, as seen in the last row of the table. In the first part (cases A1-A3), the impact of LVRT and DVS of the PV systems on ST voltage and transient stability is analyzed, as shown in detail in Section VII-C. In the second part (cases B1-B3), the impact of the active current recovery rate on the delayed voltage recovery and the frequency dynamics is investigated, as described in Section VII-D. In the third part (cases $\mathrm{C} 1-\mathrm{C} 2$ ), the impact of different local voltage control strategies is studied, as analyzed in Section VII-E. In the fourth and last part (cases D1-D4), the impact of the plantlevel voltage control of PV systems is studied, as shown in Section VII-F. The corresponding variations for the cases A$\mathrm{D}$ in Table VI, according to the control modes presented in Section IV, are highlighted in gray. All of the aforementioned cases are analyzed based on the evaluation methods described in Section VI.

\section{B. Contingencies}

The first contingency (fault 1) is a solid three-phase fault on line 4032-4044, located between the North and the Central area (see Fig. 6). The fault takes place close to bus 4044, lasts $0.1 \mathrm{~s}$ and is cleared by opening the line, which remains open.

The second contingency (fault 2) is a solid three-phase fault on one of the lines 4045-4051, located in the Central area (see Fig. 6). The fault takes place close to bus 4051 , lasts $0.1 \mathrm{~s}$ and is cleared by opening the line, which remains open.

These contingencies are selected because: i) the fault location influences PV systems, IMs, and synchronous generators; ii) the fault impedance and iii) the fault type lead to severe voltage sags; and iv) the fault duration is typical for protection systems according to international grid codes.

TABLE VI

OVERVIEW OF CASE STUDIES

\begin{tabular}{|c|c|c|c|c|c|c|c|c|c|c|c|c|c|c|}
\hline Case & 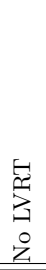 & 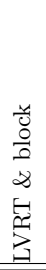 & 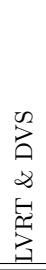 & $\begin{array}{l}\frac{\infty}{2} \\
\frac{2}{0} \\
0\end{array}$ & $\begin{array}{l}\frac{n}{a^{2}} \\
\stackrel{2}{2}\end{array}$ & $\begin{array}{l}\frac{0}{2} \\
\frac{0}{2} \\
8 \\
0\end{array}$ & $\begin{array}{l}\frac{1}{2} \\
\frac{8}{2} \\
8 \\
0 \\
\end{array}$ & 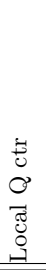 & 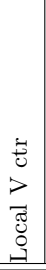 & 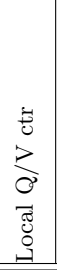 & 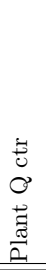 & 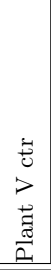 & 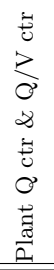 & 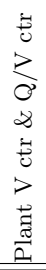 \\
\hline $\mathrm{A} 1$ & $x$ & & & & & $x$ & & $x$ & & & & & & \\
\hline $\mathrm{A} 2$ & & $\times$ & & & & $x$ & & $x$ & & & & & & \\
\hline A3 & & & $x$ & & & $x$ & & $x$ & & & & & & \\
\hline B1 & & & $x$ & $x$ & & & & $x$ & & & & & & \\
\hline B2 & & & $x$ & & $\times$ & & & $x$ & & & & & & \\
\hline B3 & & & $x$ & & & 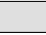 & $x$ & $x$ & & & & & & \\
\hline C1 & & & $x$ & & & $\times$ & & & $x$ & & & & & \\
\hline $\mathrm{C} 2$ & & & $x$ & & & $x$ & & & & $\times$ & & & & \\
\hline D1 & & & $x$ & & & $x$ & & $x$ & & & $x$ & & & \\
\hline D2 & & & $x$ & & & $x$ & & $x$ & & & & $x$ & & \\
\hline D3 & & & $\times$ & & & $x$ & & & & $\times$ & & & $\times$ & \\
\hline D4 & & & $x$ & & & $\times$ & & & & $\times$ & & & & $x$ \\
\hline Base & & & & & Wit & out & hoto & rolta & $c$ sys & tems & & & & \\
\hline
\end{tabular}

\section{Impact of LVRT and DVS}

This section analyzes the impact of the LVRT and DVS capability of PV systems not only on ST voltage stability and delayed voltage recovery, but also transient stability. The corresponding cases A1-A3 (see Table VI) aim at comparing the disconnection of the large-scale PV systems (A1) with the blocking (zero power) mode (A2) as well as with the LVRT and DVS capability (A3). The aforementioned control modes are described in detail in Section IV-B and the results of the analysis are shown in Fig. 9 and Table VII.

The time-domain results in response to fault 2 are gathered in Fig. 9. Due to the disconnection of PV systems (A1), large and small IMs at buses 1-5 and 51 stall because their rotor slips exceed the unstable equilibrium values. This causes ST voltage instability, as revealed by the slips $\left(s_{\mathrm{s} 2}, s_{12}\right)$ and the bus voltage $\left(V_{2}\right)$. As a result of the voltage collapse, synchronous generators g16 and g16b lose synchronism (i.e. become transient unstable), which is shown by the relative rotor angle $\left(\delta_{16}\right)$ and the voltage oscillation at the load bus $\left(V_{2}\right)$. In fact, the evolution of slips $\left(s_{\mathrm{s} 2}, s_{12}\right)$ and the rotor angle $\left(\delta_{16}\right)$ show that $\mathrm{ST}$ voltage instability and transient instability go hand in hand and appear in the same time scale. If the PV systems remain connected to the grid but reduce their active and reactive current to zero during the fault-on period (A2), ST voltage instability still takes place, as seen by the slip evolution of the large IM $\left(s_{12}\right)$ in Fig. 9. After the fault is cleared, the voltage at the load bus $\left(V_{2}\right)$ is locked at a low level. However, due to the LVRT capability of the PV systems in this case (A2), the dynamic performance of the overall power system is improved in so far as less IMs stall and no transient instability takes place. Note that due to the depressed voltage caused by the blocking mode (A2), PV systems may disconnect according to the LVRT requirements [18], but this is not considered in this investigation. The best performance is achieved with the LVRT and DVS capability of the PV systems (A3), where the latter ride through the fault and feed in an additional capacitive reactive current during the faulton period, as seen by the reactive current evolution $\left(I_{\mathrm{q}}\right)$ in Fig. 9. As a result, ST voltage and transient instabilities can be avoided, and FIDVR is significantly improved.

TABLE VII

METRICS OF ALL CONTINGENCIES AND CASES

\begin{tabular}{|c|c|c|c|c|}
\hline \multirow{2}{*}{ Case } & \multicolumn{2}{|c|}{ Fault 1 } & \multicolumn{2}{c|}{ Fault 2 } \\
\cline { 2 - 5 } & $t_{\mathrm{CCT}}[\mathrm{s}]$ & $\mathcal{V}_{4}$ of $V_{4}[-]$ & $t_{\mathrm{CCT}}[\mathrm{s}]$ & $\mathcal{V}_{2}$ of $V_{2}[-]$ \\
\hline \hline A1 & 0.02 & -0.04 & 0.05 & -0.28 \\
\hline A2 & 0.05 & -0.01 & 0.09 & -0.02 \\
\hline A3 & 0.12 & 0.66 & 0.12 & 0.64 \\
\hline \hline B1 & 0.12 & 0.22 & 0.11 & 0.58 \\
\hline B2 & 0.12 & 0.29 & 0.11 & 0.60 \\
\hline B3 & 0.12 & 0.68 & 0.12 & 0.64 \\
\hline C1 & 0.11 & -0.02 & 0.09 & 0.04 \\
\hline C2 & 0.11 & 0.49 & 0.10 & 0.55 \\
\hline \hline D1 & 0.12 & 0.65 & 0.11 & 0.63 \\
\hline D2 & 0.12 & 0.67 & 0.11 & 0.66 \\
\hline D3 & 0.11 & 0.47 & 0.10 & 0.55 \\
\hline D4 & 0.11 & 0.50 & 0.10 & 0.60 \\
\hline \hline Base & 0.15 & 0.74 & 0.12 & 0.87 \\
\hline
\end{tabular}




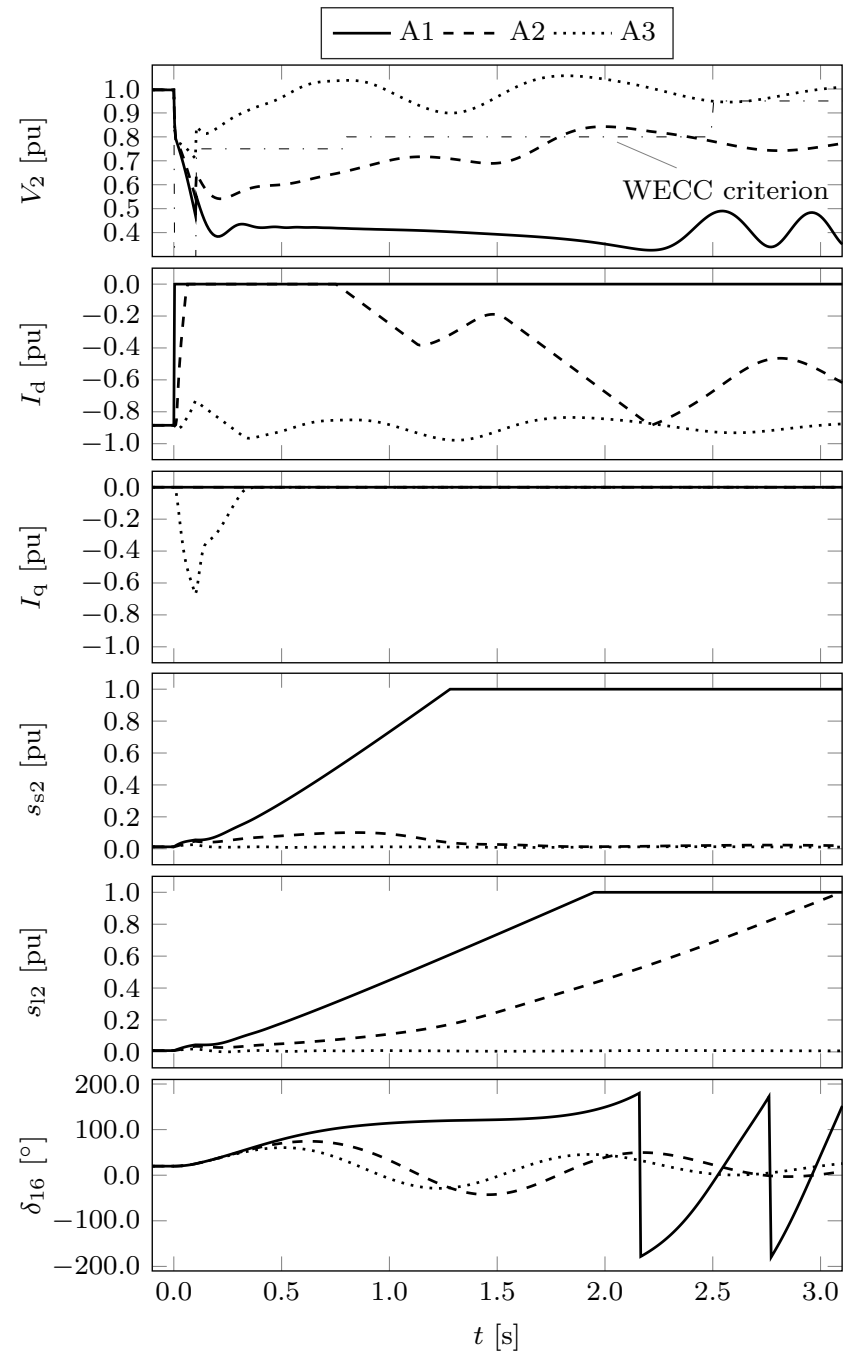

Fig. 9. Impact of LVRT and DVS (fault 2). Voltage at load bus $2\left(V_{2}\right)$. Currents of the PV system connected to bus $1042\left(I_{\mathrm{d}}, I_{\mathrm{q}}\right)$. Slips of the small and large IM load at bus $2\left(s_{\mathrm{s} 2}, s_{\mathrm{l} 2}\right)$. Rotor angle of generator g16 $\left(\delta_{16}\right)$.

The calculated metrics that evaluate the impact of LVRT and DVS are presented in Table VII and the best performance is highlighted in gray. Considering fault 2, the CCT is significantly increased from $0.05 \mathrm{~s}$ to $0.12 \mathrm{~s}$ when passing from (A1) to (A3). In the same manner, the VRI $\mathcal{V}_{2}$ at load bus 2 shows the best performance using the LVRT and DVS capability (A3) with a value of 0.64 . The lowest performance is reached if the PV systems do not have any LVRT capability (A1), with a $\mathcal{V}_{2}$ value of -0.28 . The base case without PV systems shows the best VRI due to the higher voltage support capability of synchronous generators during the fault-on period.

\section{Impact of active current recovery rate}

This section studies the impact of the active current recovery rate of PV systems on voltage recovery and frequency dynamics. The corresponding cases $\mathrm{B} 1-\mathrm{B} 3$ and $\mathrm{A} 3$ are shown in Table VI and differ by the ramp rate rrpwr of the active current, ranging from $10 \% / \mathrm{s}$ (B1) to $1000 \% / \mathrm{s}$ (B3). The details to the ramp rates can be found in Section IV-C and the results of the analysis are provided in Fig. 10 and Table VII.

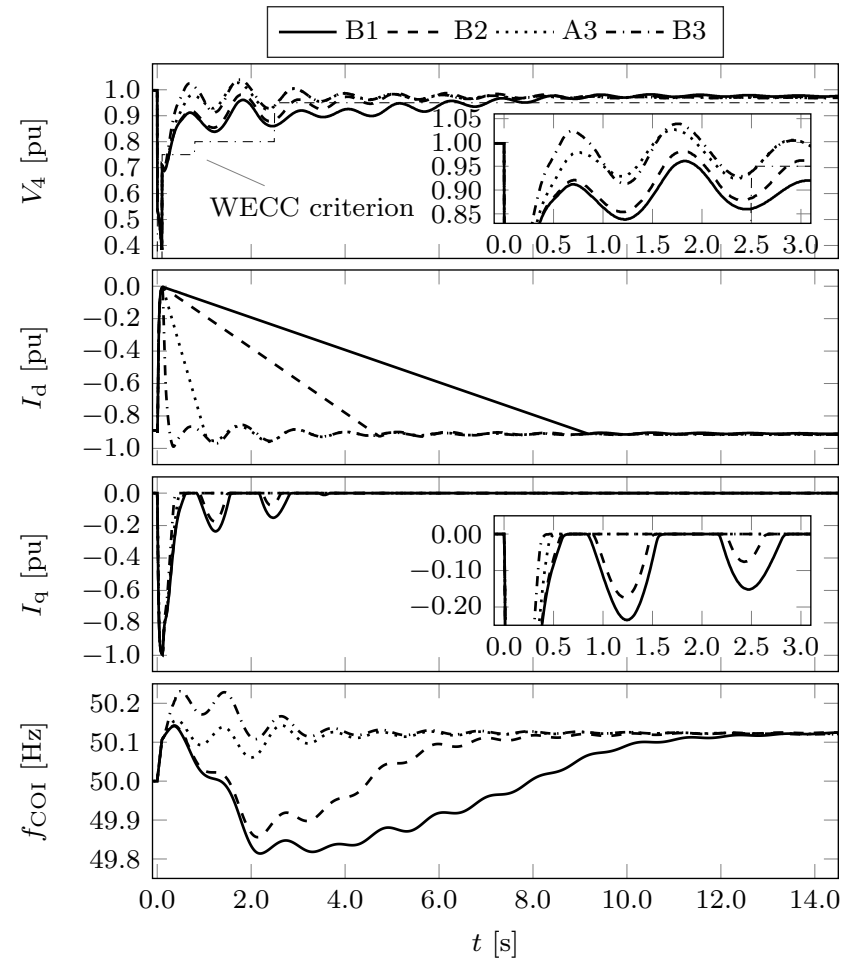

Fig. 10. Impact of active current recovery rate (fault 1). Voltage at load bus 4 $\left(V_{4}\right)$. Currents of the PV system connected to bus $1044\left(I_{\mathrm{d}}, I_{\mathrm{q}}\right)$. Frequency of the COI of the system $\left(f_{\mathrm{COI}}\right)$.

The time-domain results in response to fault 1 are shown in Fig. 10. The slow active current $\left(I_{\mathrm{d}}\right)$ recovery rates of $10 \% / \mathrm{s}$ (B1) and $20 \% / \mathrm{s}$ (B2) lead to significantly delayed voltage recoveries with voltages as low as $0.83 \mathrm{pu}$ at $1.2 \mathrm{~s}$, respectively, as shown by the load bus voltage $\left(V_{4}\right)$. Higher ramp rates, such as $100 \% / \mathrm{s}$ (A3) and $1000 \% / \mathrm{s}$ (B3) accelerate the voltage recovery, leading for instance to 0.93 pu voltage at $1.2 \mathrm{~s}$, respectively. It is noteworthy that due to the low voltages caused by the slow active current $\left(I_{\mathrm{d}}\right)$ ramping, the PV systems inject an additional reactive current $\left(I_{\mathrm{q}}\right)$ at $1.2 \mathrm{~s}$ and $2.5 \mathrm{~s}$ to support the voltage (DVS capability), even though the fault has been already cleared. At $0.6 \mathrm{~s}$, the frequency of the Center Of Inertia (COI) $\left(f_{\mathrm{COI}}\right)$ rises due to the load sensitivity (lower voltage leads to lower active power consumption). However, the slow active current $\left(I_{\mathrm{d}}\right)$ ramp rates of $10 \% / \mathrm{s}$ (B1) or $20 \% / \mathrm{s}$ (B2) lead to a frequency nadir of $49.8 \mathrm{~Hz}$ and $49.85 \mathrm{~Hz}$, respectively, at $2.2 \mathrm{~s}$. The medium recovery rate of $100 \% / \mathrm{s}$ (A3) shows the best frequency performance. However, taking voltage dynamics into account, the fast ramp rate of $1000 \% / \mathrm{s}$ (B3) is more efficient. Note that due to the fast ramp rate of $1000 \% / \mathrm{s}$ (B3) leading to frequencies higher than $50.2 \mathrm{~Hz}$, PV systems operated according to old grid code requirements might disconnect and cause a further frequency drop in the system (this is well known as the $50.2 \mathrm{~Hz}$ problem).

The calculated metrics to assess the impact of the active current recovery rate are provided in Table VII. In general, the same tendency is observed as for the time-domain results. According to the VRI $\mathcal{V}_{4}$ at load bus 4 , the best voltage recovery is achieved with the highest ramp rate of $1000 \% / \mathrm{s}$ 
(B3), with a value of 0.68 . On the other hand, a slow active current ramp rate of $10 \% / \mathrm{s}$ (B1) leads to a poor performance value with $\mathcal{V}_{4}$ of 0.22 . The CCT for fault 1 is not influenced by the different ramp rates with $t_{\mathrm{CCT}}=0.12 \mathrm{~s}$ in all cases. Note that a smaller step size for the simulations, i.e., smaller than $0.01 \mathrm{~s}$ (see Section VI-A), leads to somewhat more accurate values of the CCT. Furthermore, the base case shows a slightly better dynamic performance compared to the cases with PV systems.

\section{E. Impact of local voltage control}

This section studies the impact of local voltage control of PV systems. The corresponding cases $\mathrm{C} 1-\mathrm{C} 2$ and A3, shown in Table VI, aim at comparing different local voltage control strategies, as detailed in Section IV-D. The results of the analysis are shown in Fig. 11 and Table VII.

The time-domain results for fault 1 are depicted in Fig. 11. In the case of constant voltage control without reactive power limitation $(\mathrm{C} 1)$, the PV system tries to restore its pre-fault voltage by injecting reactive current $\left(I_{\mathrm{q}}\right)$ and therefore, reducing its active current $\left(I_{\mathrm{d}}\right)$. However, due to the rather stiff grid behavior, restoring grid voltage is impossible and the system collapses, as seen by the load bus voltage $\left(V_{4}\right)$ and the small and large IM slips $\left(s_{\mathrm{s} 2}, s_{12}\right)$, owing to the lack of active power produced by the PV systems. To overcome this problem, the coordinated reactive power/voltage control (C2) can be used, in which the second PI controller (see REEC_B_2 in Fig. 3) restores the voltage very quickly (same as (C1)), while, the first PI controller brings the reactive power slowly back to its pre-fault value. This results in a less oscillatory voltage evolution compared to the constant reactive power control (A3). Although counterintuitive, the time-domain results show that a high reactive power injection is not always the best to counteract a voltage collapse. This is in fact a matter of relative effects of active and reactive current injections.

The resulting metrics to evaluate the impact of local voltage control are given in Table VII. The best dynamic performance, with respect to delayed voltage recovery, is achieved using constant reactive power control (A3), leading to a $\mathcal{V}_{4}$ value at load bus 4 of 0.66 . As expected, using constant voltage control without reactive power limitation $(\mathrm{C} 1)$, the VRI reaches an unacceptable value when the system collapses, with $\mathcal{V}_{4}=-0.02$. The CCT for fault 1 is a little higher with constant reactive power (A3) with a value of $0.12 \mathrm{~s}$, compared to constant voltage control $(\mathrm{C} 1)$ or coordinated reactive power/voltage control (C2) with $0.11 \mathrm{~s}$, respectively. Furthermore, the base case without PV systems shows the best dynamic behavior according to the metrics presented in Table VII.

\section{F. Impact of plant-level voltage control}

The last studies focus on the impact of plant-level voltage control of PV systems on dynamics in the order of tens of seconds. The corresponding cases D1-D4 are depicted in Table VI and compare the impact of the relatively slow plant control on the voltage dynamics. The applied plant-level control modes are outlined in Section IV-E and the results of the analysis are provided in Fig. 12 and Table VII.

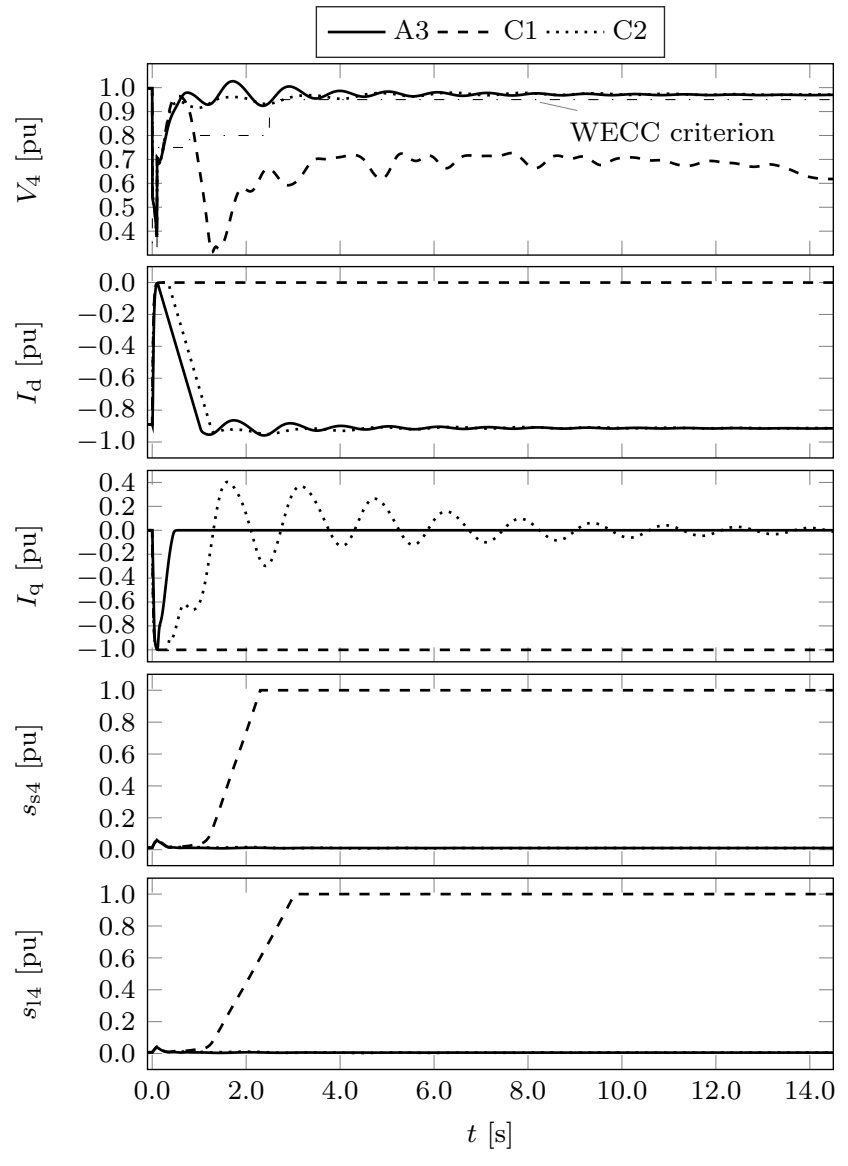

Fig. 11. Impact of local voltage control (fault 1). Voltage at load bus $4\left(V_{4}\right)$. Currents of the PV system connected to bus $1044\left(I_{\mathrm{d}}, I_{\mathrm{q}}\right)$. Slips of the small and large IM load at bus $4\left(s_{\mathrm{s} 4}, s_{14}\right)$.

The results of the time-domain simulations are provided in Fig. 12. Focusing on dynamics in the order of a few seconds, plant-level reactive power control (D1) and plant-level voltage control (D2) yield very similar behavior, as shown by the PV system currents $\left(I_{\mathrm{d}}, I_{\mathrm{q}}\right)$ and the resulting load bus voltage $\left(V_{4}\right)$. In the same time scale, plant-level reactive power control and local coordinated reactive power/voltage control (D3) as well as plant-level voltage control and local coordinated reactive power/voltage control (D4) act similarly and lead to a less oscillatory load bus voltage $\left(V_{4}\right)$ during the recovery period. On the contrary, focusing on dynamics up to $20 \mathrm{~s}$, different control mode similarities are observed, namely Plant $Q$ ctr (D1) and Plant $Q$ ctr \& $Q / V$ ctr (D3) act in a similar manner and bring the reactive power (current) back to its pre-fault value (i.e. zero), as shown by the PV system currents $\left(I_{\mathrm{d}}, I_{\mathrm{q}}\right)$ in Fig. 12. On the other hand, Plant $V$ ctr (D2) and Plant $V$ ctr \& $Q / V$ ctr (D4) try to restore the pre-fault voltage, as seen by the slow injection of reactive current $\left(I_{\mathrm{q}}\right)$. Hence, the best performance considering both voltage dynamics can be achieved with Plant $V$ ctr \& $Q / V$ ctr (D4). Note that due to the reactive power limitation and the rather slow behavior of the plant-level control, the constant voltage control modes, i.e., Plant $V$ ctr (D2) and Plant $V$ ctr \& $Q / V$ ctr (D4), can avoid the system collapse that was experienced using Local $V$ ctr (C1), as shown in Fig. 11. 

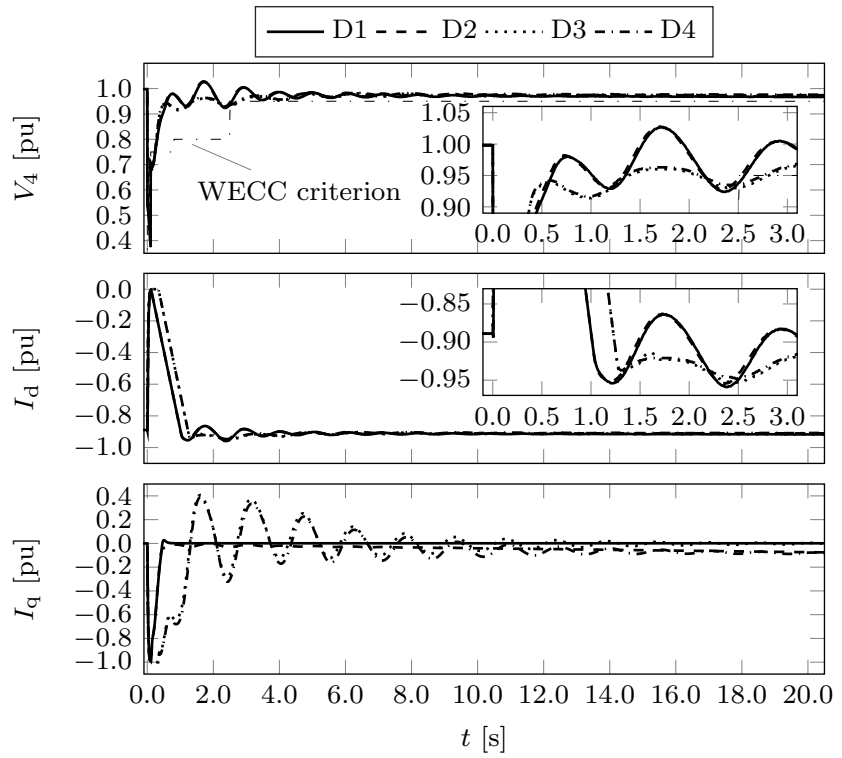

Fig. 12. Impact of plant-level voltage control (fault 1). Voltage at load bus 4 $\left(V_{4}\right)$. Currents of the PV system connected to bus $1044\left(I_{\mathrm{d}}, I_{\mathrm{q}}\right)$.

The calculated metrics to evaluate the dynamics of the plant-level voltage control are given in Table VII. The best performance according to the VRI $\mathcal{V}_{4}$ at load bus 4 is achieved with Plant $V$ ctr (D2) with a value of 0.67. Considering fault 1, Plant V ctr (D2) and Plant $Q$ ctr (D1) show the highest CCT with a value of $0.12 \mathrm{~s}$, which is still less compared to the base case.

\section{CONCLUSIONS}

This paper studies the impact of the LVRT and DVS capability, the active current recovery rate as well as local and plant-level voltage control of large-scale PV systems on ST voltage stability and recovery, and incidentally on transient and frequency stability. The studies, performed on the IEEE Nordic test system, involve WECC generic PV system models with parameter variations in accordance with international grid codes. Moreover, the CCT and a novel metric, the so-called VRI, are utilized to assess the dynamic performance.

The numerical examples show that without LVRT capability the system is ST voltage as well as transient unstable. Even the blocking mode of PV systems leads to ST voltage instability. Only the LVRT and DVS capability helps avoiding instabilities and improve FIDVR. Considering voltage and frequency dynamics, an intermediate value of the active current recovery rate of $100 \% / \mathrm{s}$ shows the best behavior. Based on voltage dynamics up to $20 \mathrm{~s}$, the best performance can be achieved using plant-level voltage control together with local coordinated reactive power/voltage control. In general, with adequate control of PV systems, the results show the improvement of power system dynamic performance to prevent incidents like those experienced recently.

Future work will consider the impact of plant-level control on long-term voltage stability. The application of Lyapunov exponents [13] to investigate ST voltage stability is also of interest.

\section{APPENDIX}

\section{KULLBACK-LEIBLER DIVERGENCE MEASURE}

Calculation of Kullback-Leibler divergence measure [23]

1) Voltage violation criterion: specifies the critical value of the KL divergence measure and, for instance, the WECC voltage violation criterion according to [24] can be used.

2) PDF of voltage sample: has the form of a normalized distribution:

$$
\mathcal{P}^{\mathrm{KL}}=\frac{1}{A} \sum_{i=1}^{L} \mathcal{P}_{i}^{\mathrm{KL}}
$$

where the number of samples in a particular sub-interval $\mathcal{P}_{i}^{\mathrm{KL}}$ is divided by the total number of samples $A$.

3) PDF of reference voltage recovery: is a Dirac-delta function with the approximation:

$$
\mathcal{P}_{\mathrm{ref}, i}^{\mathrm{KL}}=\frac{\mathrm{e}^{-\gamma\left(V_{i}-V_{\text {pre }}\right)^{2}}}{W} \quad \text { for } \quad i=1, \ldots, L
$$

where the parameter $\gamma$ controls the concentration of the density near $V_{\text {pre }}$, and $W$ denotes the normalizing factor.

4) Calculation of KL divergence measure: using the relative entropy between two PDFs:

$$
\mathcal{K}\left(\mathcal{P}^{\mathrm{KL}} \| \mathcal{P}_{\mathrm{ref}}^{\mathrm{KL}}\right)=\sum_{i=1}^{L} \mathcal{P}_{i}^{\mathrm{KL}} \ln \left(\frac{\mathcal{P}_{i}^{\mathrm{KL}}}{\mathcal{P}_{\text {ref }, i}^{\mathrm{KL}}}\right)
$$

that compares the distance between the PDF of a given voltage recovery $\mathcal{P}^{\mathrm{KL}}$ and the PDF of the reference voltage recovery $\mathcal{P}_{\text {ref }}^{\mathrm{KL}}$.

\section{Limitations of Kullback-Leibler divergence measure}

By way of example, consider the evolution of the two voltages shown in Fig. 13. One shows an ideal voltage recovery, denoted as $V_{\text {ideal }}$, which reaches its pre-fault value $V_{\text {pre }}$ immediately after the fault clearing instant $t_{\mathrm{cl}}$. On the other hand, the second evolution, denoted as $V_{\text {osc }}$, shows a delayed voltage recovery with an oscillatory behavior. It is clear that voltage recovery $V_{\text {osc }}$ is worse. Hence, the KL divergence measure for the ideal voltage recovery $\mathcal{K}_{\text {ideal }}$ is expected to be smaller. However, from the calculated divergence measures using $\gamma=450$ and the same values shown in Table IV, yield $\mathcal{K}_{\text {ideal }}=1.94$ and $\mathcal{K}_{\text {osc }}=0.84$. As $\mathcal{K}_{\text {ideal }}$ is higher than $\mathcal{K}_{\text {osc }}$, the former suggests a lower quality of recovery, which is contradictory. This is due to the fact that the distance of the PDF of the delayed voltage recovery $\mathcal{P}_{\text {osc }}^{\mathrm{KL}}$ to the PDF of the reference voltage recovery $\mathcal{P}_{\text {ref }}^{\mathrm{KL}}$ is shorter, i.e., $\mathcal{K}\left(\mathcal{P}_{\text {osc }}^{\mathrm{KL}} \| \mathcal{P}_{\text {ref }}^{\mathrm{KL}}\right)<\mathcal{K}\left(\mathcal{P}_{\text {ideal }}^{\mathrm{KL}} \| \mathcal{P}_{\text {ref }}^{\mathrm{KL}}\right)$. This gives the motivation to define an improved metric, namely the VRI.

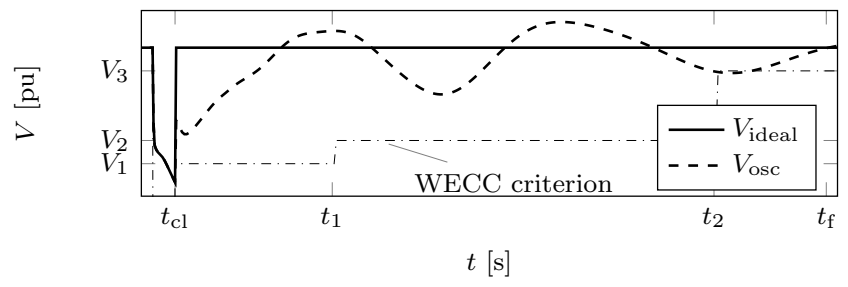

Fig. 13. Ideal and oscillatory voltage recovery. 


\section{REFERENCES}

[1] North American Electric Reliability Corporation (NERC). (2017, Jun.) 1200 MW Fault Induced Solar Photovoltaic Resource Interruption Disturbance Report - Southern California 8/16/2016 Event. [Online]. Available: http://www.nerc.com/pa/rrm/ea/1200_ MW_Fault_Induced_Solar_Photovoltaic_Resource_/1200_MW_Fault_ Induced_Solar_Photovoltaic_Resource_Interruption_Final.pdf

[2] Australian Energy Market Operator (AEMO). (2017, Mar.) Black System South Australia 28 September 2016. [Online]. Available: https://www.aemo.com.au/-/media/Files/Electricity/NEM/ Market_Notices_and_Events/Power_System_Incident_Reports/2017/ Integrated-Final-Report-SA-Black-System-28-September-2016.pdf

[3] G. Lammert, J. C. Boemer, D. Premm, O. Glitza, L. D. Pabón Ospina, D. Fetzer, and M. Braun, "Impact of Fault Ride-Through and Dynamic Reactive Power Support of Photovoltaic Systems on Short-Term Voltage Stability," in 2017 IEEE PowerTech, Manchester, Jun. 2017.

[4] R. Shah, N. Mithulananthan, R. Bansal, and V. Ramachandaramurthy, "A Review of Key Power System Stability Challenges for Large-Scale PV Integration," Renewable and Sustainable Energy Reviews, vol. 41, no. Supplement C, pp. 1423 - 1436, 2015.

[5] S. Eftekharnejad, V. Vittal, G. T. Heydt, B. Keel, and J. Loehr, "Impact of Increased Penetration of Photovoltaic Generation on Power Systems," IEEE Trans. on Power Systems, vol. 28, no. 2, pp. 893-901, May 2013.

[6] B. Tamimi, C. Cañizares, and K. Bhattacharya, "System Stability Impact of Large-Scale and Distributed Solar Photovoltaic Generation: The Case of Ontario, Canada," IEEE Trans. on Sustainable Energy, vol. 4, no. 3, pp. 680-688, Jul. 2013

[7] B. Weise, "Impact of K-Factor and Active Current Reduction During Fault-Ride-Through of Generating Units Connected via Voltage-Sourced Converters on Power System Stability," IET Renewable Power Generation, vol. 9, no. 1, pp. 25-36, 2015.

[8] J. Quintero, V. Vittal, G. T. Heydt, and H. Zhang, "The Impact of Increased Penetration of Converter Control-Based Generators on Power System Modes of Oscillation," IEEE Trans. on Power Systems, vol. 29 no. 5, pp. 2248-2256, Sep. 2014

[9] P. Pourbeik, S. Soni, A. Gaikwad, and V. Chadliev, "Providing Primary Frequency Response from Photovoltaic Power Plants," in CIGRE Symposium, Dublin, 2017

[10] K. Kawabe, Y. Ota, A. Yokoyama, and K. Tanaka, "Novel Dynamic Voltage Support Capability of Photovoltaic Systems for Improvement of Short-Term Voltage Stability in Power Systems," IEEE Trans. on Power Systems, vol. 32, no. 3, pp. 1796-1804, May 2017.

[11] P. Kundur, J. Paserba, V. Ajjarapu, G. Andersson, A. Bose, C. Cañizares, N. Hatziargyriou, D. Hill, A. Stankovic, C. Taylor, T. Van Cutsem, and V. Vittal, "Definition and Classification of Power System Stability IEEE/CIGRE Joint Task Force on Stability Terms and Definitions," IEEE Trans. on Power Systems, vol. 19, no. 3, pp. 1387-1401, Aug. 2004.

[12] T. Van Cutsem and C. Vournas, Voltage Stability of Electric Power Systems. Springer, 2008.

[13] S. Dasgupta, M. Paramasivam, U. Vaidya, and V. Ajjarapu, "Real-Time Monitoring of Short-Term Voltage Stability Using PMU Data," IEEE Trans. on Power Systems, vol. 28, no. 4, pp. 3702-3711, Nov. 2013.

[14] C. W. Taylor, Power System Voltage Stability. EPRI Power System Engineering Series. McGraw Hill, 1994.

[15] Western Electricity Coordinating Council (WECC) Renewable Energy Modeling Task Force. (2012, Sep.) WECC Solar PV Dynamic Model Specification. [Online]. Available: https://www.wecc.biz/Reliability/ WECCSolarPVDynamicModelSpecification-September2012.pdf

[16] —. (2014, Apr.) WECC Solar Plant Dynamic Modeling Guidelines. [Online]. Available: https://www.wecc.biz/Reliability/ WECCSolarPlantDynamicModelingGuidelines.pdf

[17] G. Lammert, L. D. Pabón Ospina, P. Pourbeik, D. Fetzer, and M. Braun, "Implementation and Validation of WECC Generic Photovoltaic System Models in DIgSILENT PowerFactory," in 2016 IEEE Power \& Energy Society General Meeting, Boston, Jul. 2016.

[18] VDE-AR-N 4120, "Technical Requirements for the Connection and Operation of Customer Installations to the High-Voltage Network (TCC High-Voltage)," Jan. 2015.

[19] National Grid, "Guidance Notes - Power Park Modules," Issue 3, 2012.

[20] IEEE Task Force on Test Systems for Voltage Stability and Security Assessment, "Test Systems for Voltage Stability Analysis and Security Assessment," PES-TR19, Tech. Rep., Aug. 2015.

[21] L. D. Pabón Ospina, A. Correa, and G. Lammert, "Implementation and Validation of the Nordic Test System in DIgSILENT PowerFactory," in 2017 IEEE PowerTech, Manchester, Jun. 2017.

[22] DIgSILENT GmbH, “DIgSILENT PowerFactory,” Version 2018.
[23] S. Dasgupta, M. Paramasivam, U. Vaidya, and V. Ajjarapu, "EntropyBased Metric for Characterization of Delayed Voltage Recovery," IEEE Trans. on Power Systems, vol. 30, no. 5, pp. 2460-2468, Sep. 2015.

[24] D. J. Shoup, J. J. Paserba, and C. W. Taylor, "A Survey of Current Practices for Transient Voltage Dip/Sag Criteria Related to Power System Stability," in IEEE PES Power Systems Conference and Exposition, New York, Oct. 2004.

[25] M. Paramasivam, S. Dasgupta, V. Ajjarapu, and U. Vaidya, "Contingency Analysis and Identification of Dynamic Voltage Control Areas," IEEE Trans. on Power Systems, vol. 30, no. 6, pp. 2974-2983, Nov. 2015.

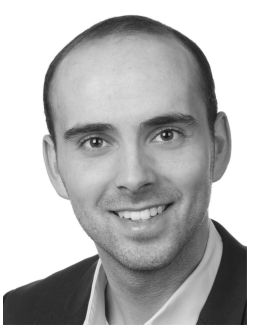

Gustav Lammert received the B.Eng. degree in electrical engineering from the Baden-Wuerttemberg Cooperative State University, Mannheim, Germany, in 2010 and the M.Sc. degree in renewable energies and energy efficiency from the University of Kassel, Kassel, Germany, in 2013. He is now a Ph.D. student at the Dept. of Energy Management and Power System Operation, University of Kassel, Kassel, Germany. His fields of interest are modelling of PV systems, power system dynamics and stability.

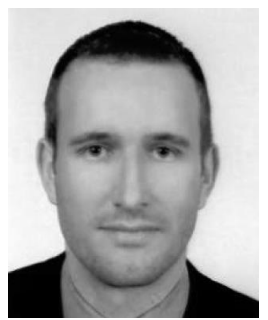

Daniel Premm received the Diploma degree in electrical engineering from Trier University of Applied Sciences, Trier, Germany, in 2008 and the M.Sc. degree in renewable energy systems from the University of Hagen, Hagen, Germany, in 2017. He is currently a System Development Engineer with focus on power plant design and system analysis, SMA Solar Technology AG, Niestetal, Germany.

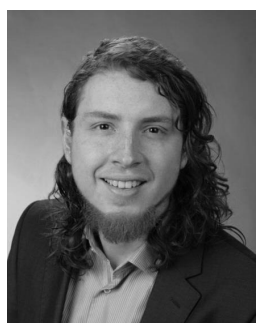

Luis David Pabón Ospina received the B.Sc. and M.Sc. degrees in electrical engineering from Universidad Pontificia Bolivariana, Medellín, Colombia, in 2011 and 2014, respectively. He received his M.Eng. degree from the University of Kempten, Kempten, Germany, in 2014. He is currently working toward the Ph.D. degree at the Dept. of Power System Control and Dynamics, Fraunhofer Institute for Energy Economics and Energy System Technology (IEE), Kassel, Germany.

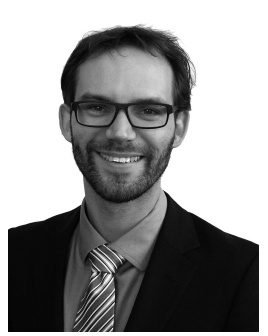

Jens C. Boemer received the Diploma degree in electrical engineering from Technical University of Dortmund, Dortmund, Germany, in 2005 and the Ph.D. from Delft University of Technology, Delft, the Netherlands, in 2016. He is now a Principal Technical Leader at the Dept. of Grid Operations and Planning, Modeling and Simulation, Electric Power Research Institute (EPRI), Palo Alto, USA. His field of interest is the grid integration of renewable energy resources with the focus on power system stability.

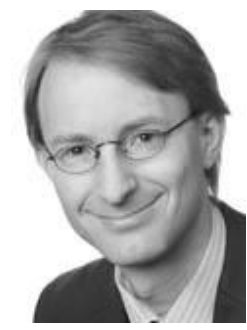

Martin Braun received Diploma degrees in electrical engineering and business administration from the University of Stuttgart, Stuttgart, Germany in 2005 and the Ph.D. in engineering from the University of Kassel, Kassel, Germany, in 2008. Since 2012 he is Full Professor at the Dept. of Energy Management and Power System Operation at the University of Kassel, and head of the Dept. of Grid Planning and Operation at the Fraunhofer Institute for Energy Economics and Energy System Technology (IEE),

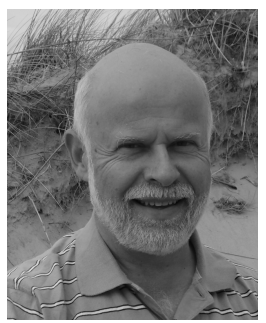

Thierry Van Cutsem received the M.Sc. and Ph.D. degrees from the University of Liège, Liège, Belgium, in 1979 and 1984, respectively. He is currently a Research Director of the Fund for Scientific Research (FNRS) and an Adjunct Professor at the Dept. of Electrical Engineering and Computer Science at the same university. His research interests include power system dynamics, security, monitoring, control, and simulation. He served as Chair of the IEEE Power System Dynamic Performance Committee. 